\title{
Inflammation Cytokine Expression and Association With Clinical Outcomes in Critically III Patients After Major Surgery: A Retrospective Cohort Study
}

\section{Yamin Yan}

Zhongshan Hospital Fudan University

\section{Xiaorong Wang}

Zhongshan Hospital Fudan University

\section{Yan $\mathrm{Hu}$}

Zhongshan Hospital Fudan University

\section{Zhenghong Yu}

Shanghai Stomatological Disease Center

\section{Yingjia Tang}

Zhongshan Hospital Fudan University

\section{Jingjing Li}

Zhongshan Hospital Fudan University

Jinghua Mei

Zhongshan Hospital Fudan University

\section{Wenyan Pan ( $\triangle$ panwenyan1987@126.com )}

Zhongshan Hospital Fudan University https://orcid.org/0000-0003-0624-0390

\section{Yuxia Zhang}

Zhongshan Hospital Fudan University

\section{Research}

Keywords: Cytokine expression, major surgery, critical ill patients, mortality, SICU readmission

Posted Date: June 4th, 2020

DOl: https://doi.org/10.21203/rs.3.rs-32628/v1

License: (c) (1) This work is licensed under a Creative Commons Attribution 4.0 International License. Read Full License 


\section{Abstract}

Background The associations of serum cytokine levels and critically ill patient outcomes after major surgery remain unclear. The use of cytokine markers to predict outcomes in critically ill patients is controversial.

Objective To determine the levels of IL-1 $\beta$, IL-2, IL-6, IL-8, IL-10, TNF- $a$ and procalcitonin in critical surgical ICU(SICU) patients and evaluate their associations with patient outcome and clinical significance.

Methods This was a retrospective cohort study of consecutive patients admitted to the SICU in Zhongshan Hospital, Fudan University. The program ran from January 1, 2018, to June 30, 2019. The levels of IL-1 $\beta$, IL-2, IL-6, IL-8, IL-10, TNF-a and procalcitonin were detected, and their relationship with patient outcomes was investigated. The primary outcome was in-hospital mortality, compared by a multivariable logistic regression analysis among the survivors and nonsurvivors.

Results Overall, 5,257 patients were included in this study for their first SICU admission; 5,099 patients survived, 158 patients died, and the mortality rate was $3.0 \%(158 / 5,257)$. Univariate and multivariate analyses showed that nonsurvivors had increased levels of IL-1 $(\mathrm{OR}=1.855, \mathrm{P}=0.000)$ and IL-2(OR=1.51, $\mathrm{P}=0.000$ ) compared with survivors. In addition, 196 patients(3.7\%) were readmitted to the SICU, and data from 187 patients were collected. Of these, 161 patients survived, and 26 patients died; the mortality rate was $13.9 \%(26 / 187)$, which was much higher than that of the first round of patients. The level of IL-2 significantly influenced SICU readmission(OR=3.921, $\mathrm{P}=0.000)$. For the third round of SICU admission, 10 patients were included, 7 patients survived, and 3 patients died; the mortality rate was $30.0 \%(3 / 10)$. Furthermore, older age, longer time of SICU stay, and higher rate of mechanical ventilation and CRRT were associated with patient death.

Conclusions High levels of cytokines may be risk factors for mortality and SICU readmission in critically ill patients who receive major surgery. Further work is still needed to determine which unmeasured characteristics and therapies may contribute to the increased risk observed.

\section{Background}

Despite advances in the management of critically ill patients, mortality among the ICU population remains high. Outcome prediction could provide useful information regarding therapeutic decision making and guide resource allocation[1]. However, the prediction of ICU mortality is still challenging.

There is mounting evidence indicating that cytokines are implicated in the pathogenesis of critical illness[2]. The occurrence and development of illness are closely associated with the human immune system. Cytokines play an important role in regulating the immunologic response, which mediates the severity of disease and associated complications. For example, IL-2, an important component of cellular immunity that promotes the differentiation of activated $T$ cells, directly affects the function of immune cells and the local immune status[3]. In critically ill states, high levels of inflammatory cytokines are 
positively related to the severity of underlying disease, and their persistence in the circulation and intense activation of inflammatory mediators play important roles in the development of multiple organ failure (MOF) $[4,5]$. The serum level of IL-6 is associated with clinical outcome or organ dysfunction severity in critically ill patients $[6,7]$ and may be useful for predicting the development of acute kidney injury. In sepsis, the balance between tumor necrosis factor-alpha (TNF-a) and interleukin-10 (IL-10) determines clinical outcome[8].

Cytokine responses in critically ill patients who have just undergone surgery have been less investigated. In particular, the associations between cytokine expression and outcomes in such patients have not been well defined. A variety of pro- and anti-inflammatory mediators seem to be involved in the pathogenesis of systemic inflammatory response syndrome (SIRS)[9] after operation; however, the pattern of their evolution during the course of SIRS remains largely unexplored. One study reported that early after the end of the operation, IL-6, IL-8 and IL-10 were elevated compared to their baseline levels. These increases were followed by increases in TNF-a, which was increased after one day. Moreover, it was shown that the balance between TNF- $a$ and IL-10 may be related to the occurrence of postoperative complications[10].

The purpose of this study was to identify the expression levels of IL-1 $\beta$, IL-2, IL-6, IL-8, IL-10, TNF-a and procalcitonin in critical surgical ICU (SICU) patients and to investigate whether the expression level is associated with key clinical outcomes such as ICU readmission and mortality risk in SICU patients. Furthermore, in readmitted patients, we evaluated the correlation between cytokine levels and patient mortality and the rate of a third readmission to the SICU.

\section{Materials And Methods}

\section{Setting and patients}

Before the analysis, all the patients' information was anonymized and deidentified. All participants provided written informed consent.

This study was conducted in the SICU in a teaching hospital that has 28 beds and included all mechanically ventilated, critically ill patients who received surgery. Adult ( $\geq 18$ years of age) critically ill patients who recently received surgery were enrolled. Patients were excluded if baseline data were missing or data on serum cytokine expression levels on SICU admission were lacking. In total, 5,257 patients were enrolled.

\section{Data Collection}

We extracted the following data of critically ill patients who received surgery: demographics, diagnosis, surgical status and urgency (emergency surgery or elective surgery), cytokine level (IL-1 $\beta$, IL-2, IL-6, IL-8, IL-10, TNF- $\alpha$ and procalcitonin), type of organ support (i.e., mechanical ventilation or continuous renal replacement therapy (CRRT)), etc. The data of age, sex, admitting diagnosis, and severity of illness were 
recorded upon entry into the SICU. Data were collected daily by trained data collectors from the time of SICU admission to death or SICU discharge. Data included in this study were collected between January 1, 2018, and June 31, 2019.

These analyses were carried out retrospectively, and informed consent was required. The procedure mentioned in this study was in accordance with the standards of the Ethics Committee for Human Experimentation of Zhongshan Hospital, Fudan University.

\section{Cytokine Measurements}

Blood samples were collected within $24 \mathrm{~h}$ of SICU admission to check the expression levels of IL-1 $\beta$, IL-2, IL-6, IL-8, IL-10, TNF-a and procalcitonin. Concentrations of these parameters were measured in duplicate after staining with monoclonal antibodies and passage through IMMULITE 1000 Immunoassay System (Siemens, Berlin). Normal levels of detection were lower than $5 \mathrm{pg} / \mathrm{ml}$ for IL-1 $3,223-710 \mathrm{U} / \mathrm{ml}$ for IL-2, lower than $3.4 \mathrm{pg} / \mathrm{ml}$ for IL-6, lower than $62 \mathrm{pg} / \mathrm{ml}$ for IL-8, lower than $9.1 \mathrm{pg} / \mathrm{ml}$ for IL-10, lower than $8.1 \mathrm{pg} / \mathrm{ml}$ for TNF-a and lower than $0.5 \mathrm{ng} / \mathrm{ml}$ for procalcitonin.

\section{Outcomes}

The primary outcome was hospital mortality. Secondary outcomes included several SICU-specific outcomes: SICU readmission, SICU length of stay, receipt of tracheostomy, total days of organ support, receipt and duration of mechanical ventilation at any time during SICU admission, and receipt and duration of renal replacement therapy. Mechanical ventilation was defined as positive pressure ventilation via an endotracheal tube or tracheostomy. Renal replacement therapy included all forms of continuous renal replacement therapy and intermittent hemodialysis.

\section{Statistical analysis}

All statistical analyses were conducted using the statistical package SPSS for Windows (Version 22.0, SPSS, Chicago, IL). Data are presented as medians and interquartile ranges (IQRs) for data that did not follow a normal distribution and as the means \pm standard deviations (SDs) for parameters that followed a normal distribution. Between-group comparisons were performed with Student's t-test or the MannWhitney-Wilcoxon test. Outcome frequency was examined using descriptive statistics. The chi-square test or Fisher's exact test was used for univariate analysis of the collected variables. Multilevel mixed-effects logistic regression analysis was performed to examine the association between cytokine expression level and hospital mortality. The odds ratios (ORs) and 95\% confidence intervals (Cl) are reported. Those variables that were significantly related to outcome were also included in a multivariate logistic regression analysis to examine their independent effect on outcome. Kaplan-Meier survival curves were plotted and compared using the log-rank test for overall survival analysis. Time to events (death or 
readmission) was defined as the time from SICU admission to the event being analyzed. A P value $<0.05$ was considered to show statistical significance.

\section{Results}

Demographics and clinical characteristics A total of 5,257 patients were included in this study. The mean age of the nonsurvivors was $69.7 \pm 17.1$ years, which was older than that of survivors. Overall, 5,099 patients survived, and 158 patients died, yielding a mortality rate of 3.0\%. Death occurred 1-228 days following SICU admission. In the nonsurvivor group, the length of SICU stay and length of mechanical ventilation were much longer than those in the survivor group; the rates of SICU readmission, mechanical ventilation, CRRT, tracheotomy and emergency surgery were much higher in the nonsurvivor group than the survivor group. According to the department, the highest mortality rate occurred in the general surgery department (6.2\%), and the lowest occurred in the thoracic surgery department (0.5\%). The demographics and clinical characteristics of survivors and nonsurvivors are shown in Table 1. 
Table 1

Baseline patient characteristics $(n=5,257)$

\begin{tabular}{|c|c|c|c|c|}
\hline \multirow[t]{3}{*}{ Variables } & \multicolumn{2}{|c|}{ Outcome $(n=5,257)$} & \multirow[t]{3}{*}{$t / z / \chi^{2}$} & \multirow[t]{3}{*}{$P$ value } \\
\hline & Nonsurvivors & Survivors & & \\
\hline & $(n=158)$ & $(n=5,099)$ & & \\
\hline Age (year) & $69.7 \pm 17.1$ & $62.1 \pm 16.2$ & 5.748 & 0.000 \\
\hline \multicolumn{3}{|l|}{ Sex, No. (\%) } & \multirow[t]{3}{*}{0.611} & \multirow[t]{3}{*}{0.434} \\
\hline Male & $99(3.2 \%)$ & $3,037(96.8 \%)$ & & \\
\hline Female & $59(2.8 \%)$ & $2,062(97.2 \%)$ & & \\
\hline Length of SICU stay (h) & $338.5 \pm 649.6$ & $63.7 \pm 144.7$ & 15.575 & 0.000 \\
\hline \multicolumn{3}{|l|}{ Length of SICU stay } & \multirow[t]{3}{*}{326.208} & \multirow[t]{3}{*}{0.000} \\
\hline$\otimes 7$ days & $65(19.2 \%)$ & $273(80.8 \%)$ & & \\
\hline$\leq 7$ days & $93(1.9 \%)$ & $4,826(98.1 \%)$ & & \\
\hline \multicolumn{3}{|l|}{ SICU readmission } & \multirow[t]{3}{*}{88.864} & \multirow[t]{3}{*}{0.000} \\
\hline Yes & $28(14.3 \%)$ & $168(85.7 \%)$ & & \\
\hline No & $130(2.6 \%)$ & $4,931(97.4 \%)$ & & \\
\hline \multicolumn{3}{|l|}{ Mechanical ventilation } & \multirow[t]{3}{*}{394.924} & \multirow[t]{3}{*}{0.000} \\
\hline Yes & $141(11.5 \%)$ & $1,087(88.5 \%)$ & & \\
\hline No & $17(0.4 \%)$ & $4,012(99.6 \%)$ & & \\
\hline Length of mechanical ventilation (h) & $396.0 \pm 774.7$ & $109.7 \pm 311.9$ & 8.262 & 0.000 \\
\hline \multicolumn{5}{|l|}{ Tracheotomy } \\
\hline Yes & 49 (19.4\%) & $203(80.6 \%)$ & \multirow[t]{2}{*}{245.402} & \multirow[t]{2}{*}{0.000} \\
\hline No & $109(2.2 \%)$ & $4,896(97.8 \%)$ & & \\
\hline \multicolumn{3}{|l|}{ CRRT } & \multirow[t]{3}{*}{315.779} & \multirow[t]{3}{*}{0.000} \\
\hline Yes & $45(25.4 \%)$ & $132(74.6 \%)$ & & \\
\hline No & $113(2.2 \%)$ & $4,967(97.8 \%)$ & & \\
\hline Length of CRRT (h) & $87.6 \pm 83.0$ & $70.7 \pm 94.8$ & 1.071 & 0.286 \\
\hline Emergency surgery & & & 145.588 & 0.000 \\
\hline
\end{tabular}

SICU surgical intensive care unit, TNF-a tumor necrosis factor-alpha, IL interleukin, CRRT continuous renal replacement therapy 


\begin{tabular}{|c|c|c|c|c|}
\hline \multirow[t]{3}{*}{ Variables } & \multicolumn{2}{|c|}{ Outcome $(n=5,257)$} & \multirow[t]{3}{*}{$t / z / x^{2}$} & \multirow[t]{3}{*}{$P$ value } \\
\hline & Nonsurvivors & Survivors & & \\
\hline & $(n=158)$ & $(n=5,099)$ & & \\
\hline Yes & $149(9.6 \%)$ & $1,406(90.4 \%)$ & & \\
\hline No & $8(0.2 \%)$ & $3,679(99.8 \%)$ & & \\
\hline Not known & $1(6.7 \%)$ & $14(93.3 \%)$ & & \\
\hline \multicolumn{3}{|l|}{ Department } & \multirow[t]{8}{*}{71.338} & \multirow[t]{8}{*}{0.000} \\
\hline General surgery & $76(6.2 \%)$ & $1,152(93.8 \%)$ & & \\
\hline Thoracic surgery & $6(0.5 \%)$ & $1,267(99.5 \%)$ & & \\
\hline Orthopedics & $11(2.6 \%)$ & $406(97.4 \%)$ & & \\
\hline Neurosurgery & $26(3.3 \%)$ & $772(96.7 \%)$ & & \\
\hline Endovascular surgery & $23(4.1 \%)$ & $535(95.9 \%)$ & & \\
\hline Urologic surgery & $4(1.3 \%)$ & $313(98.7 \%)$ & & \\
\hline Others & $12(1.8 \%)$ & $654(98.2 \%)$ & & \\
\hline
\end{tabular}

Cytokine levels were increased in nonsurvivors To determine whether cytokine levels were associated with hospital mortality, we assessed the difference between survivors and nonsurvivors. Nonsurvivors had increased levels of IL-1 $\beta$, IL-2, IL-6, IL-8, IL-10, TNF-a and procalcitonin compared with survivors (Table 2). Univariate and multivariate analyses showed that there was a significant correlation between the levels of IL-1 $\beta$ and IL-2 and outcomes, but no correlation was noted between IL-6, IL-8, IL-10, TNF-a and procalcitonin expression and outcomes in the patients (Table 3). 
Table 2

Cytokine levels in survivors and nonsurvivors $(n=5,257)$

\begin{tabular}{|c|c|c|c|c|}
\hline \multirow[t]{3}{*}{ Cytokines } & \multicolumn{2}{|c|}{ Outcome $(n=5,257)$} & \multirow[t]{3}{*}{ z } & \multirow[t]{3}{*}{$P$ value } \\
\hline & Nonsurvivors & Survivors & & \\
\hline & $(n=158)$ & $(n=5,099)$ & & \\
\hline \multicolumn{3}{|l|}{$\mathrm{IL}-1 \beta(\mathrm{pg} / \mathrm{ml})$} & \multirow[t]{4}{*}{-10.415} & \multirow[t]{4}{*}{0.000} \\
\hline Normal $(\leq 5)$ & $128(2.5 \%)$ & $4,922(97.5 \%)$ & & \\
\hline $5 \sim 10$ & $12(9.0 \%)$ & $121(91.0 \%)$ & & \\
\hline$\geq 10$ & $18(24.3 \%)$ & $56(75.7 \%)$ & & \\
\hline \multicolumn{3}{|l|}{ IL-2 (U/ml) } & \multirow[t]{6}{*}{-14.487} & \multirow[t]{6}{*}{0.000} \\
\hline Normal (223-710) & $31(1.0 \%)$ & $3,181(99.0 \%)$ & & \\
\hline $710-1,000$ & $22(2.3 \%)$ & $922(97.7 \%)$ & & \\
\hline $1,000-2,000$ & $51(5.8 \%)$ & $786(94.2 \%)$ & & \\
\hline $2,000-3,000$ & $27(16.3 \%)$ & $139(83.7 \%)$ & & \\
\hline$\geq 3,000$ & $27(27.6 \%)$ & $71(72.4 \%)$ & & \\
\hline \multicolumn{3}{|l|}{ IL-6 (pg/ml) } & \multirow[t]{6}{*}{-8.93} & \multirow[t]{6}{*}{0.000} \\
\hline Normal ( $₫ 3.4)$ & $3(0.8 \%)$ & $387(99.2 \%)$ & & \\
\hline $3.4-50$ & $46(1.6 \%)$ & $2,756(98.4 \%)$ & & \\
\hline $50-100$ & $29(3.7 \%)$ & $762(96.3 \%)$ & & \\
\hline $100-200$ & $22(3.7 \%)$ & $578(96.3 \%)$ & & \\
\hline$\geq 200$ & $58(8.6 \%)$ & $616(91.4 \%)$ & & \\
\hline \multicolumn{3}{|l|}{ IL-8 (pg/ml) } & \multirow[t]{6}{*}{-10.964} & \multirow[t]{6}{*}{0.000} \\
\hline Normal $(<62)$ & $87(1.9 \%)$ & $4,378(98.1 \%)$ & & \\
\hline $62-100$ & $22(6.9 \%)$ & 295 (93.1\%) & & \\
\hline $100-150$ & $13(7.6 \%)$ & 159 (92.4\%) & & \\
\hline $150-200$ & $5(5.2 \%)$ & $92(94.8 \%)$ & & \\
\hline$\geq 200$ & $31(15.0 \%)$ & $175(85.0 \%)$ & & \\
\hline \multicolumn{3}{|l|}{ IL-10 (pg/ml) } & \multirow[t]{2}{*}{-9.474} & \multirow[t]{2}{*}{0.000} \\
\hline Normal $(<9.1)$ & $79(1.9 \%)$ & $3,994(98.1 \%)$ & & \\
\hline
\end{tabular}




\begin{tabular}{|c|c|c|c|c|}
\hline $9.1-20$ & $25(3.6 \%)$ & 675 (96.4\%) & & \\
\hline $20-50$ & $19(6.4 \%)$ & 278 (93.6\%) & & \\
\hline $50-100$ & $11(12.5 \%)$ & 77 (87.5\%) & & \\
\hline$\geq 100$ & $24(24.2 \%)$ & $75(75.8 \%)$ & & \\
\hline TNF-a (pg/ml) & & & -10.544 & 0.000 \\
\hline Normal $(<8.1)$ & $21(0.9 \%)$ & 2,330 (99.1\%) & & \\
\hline $8.1-15$ & $47(2.8 \%)$ & $1,642(97.2 \%)$ & & \\
\hline $15-30$ & $40(4.8 \%)$ & 797 (95.2\%) & & \\
\hline $30-50$ & $26(10.9 \%)$ & $212(89.1 \%)$ & & \\
\hline$\geq 50$ & $24(16.9 \%)$ & $118(83.1 \%)$ & & \\
\hline Procalcitonin (ng/ml) & & & -10.938 & 0.000 \\
\hline Normal $(<0.5)$ & $72(1.9 \%)$ & $3,706(98.1 \%)$ & & \\
\hline $0.5-2$ & 27 (3.4\%) & 766 (96.6\%) & & \\
\hline $2-5$ & $17(5.7 \%)$ & $281(94.3 \%)$ & & \\
\hline $5-10$ & $13(6.5 \%)$ & $188(93.5 \%)$ & & \\
\hline$\geq 10$ & $29(15.5 \%)$ & 158 (84.5\%) & & \\
\hline
\end{tabular}


Table 3

Analysis of cytokine levels in relation to outcomes of patients

\begin{tabular}{|llllll|}
\hline Variables & $\boldsymbol{\beta}$ & SE & P value & OR & 95\% CI \\
\hline Univariate analysis & & & & & \\
\hline Sex & -0.183 & 0.214 & 0.392 & 0.833 & $(0.547,1.267)$ \\
\hline Length of SICU stay & -0.949 & 0.219 & 0.000 & 0.387 & $(0.252,0.595)$ \\
\hline SICU readmission & 0.933 & 0.286 & 0.001 & 2.543 & $(1.452,4.454)$ \\
\hline Mechanical ventilation & 2.464 & 0.312 & 0.000 & 11.75 & $(6.378,21.649)$ \\
\hline CRRT & 0.911 & 0.268 & 0.001 & 2.487 & $(1.472,4.202)$ \\
\hline Department & 0.064 & 0.08 & 0.430 & 1.066 & $(0.91,1.248)$ \\
\hline IL1 & 0.604 & 0.157 & 0.000 & 1.83 & $(1.345,2.488)$ \\
\hline IL2 & 0.406 & 0.1 & 0.000 & 1.5 & $(1.234,1.824)$ \\
\hline IL6 & -0.119 & 0.1 & 0.231 & 0.887 & $(0.73,1.079)$ \\
\hline IL8 & 0.018 & 0.095 & 0.848 & 1.018 & $(0.846,1.226)$ \\
\hline IL10 & -0.06 & 0.11 & 0.584 & 0.942 & $(0.76,1.168)$ \\
\hline TNF-a & 0.077 & 0.114 & 0.502 & 1.08 & $(0.863,1.351)$ \\
\hline Procalcitonin & 0.097 & 0.081 & 0.231 & 1.102 & $(0.940,1.292)$ \\
\hline Multivariate analysis & 0.618 & 0.127 & 0.000 & 1.855 & $(1.446,2.38)$ \\
\hline Length of SICU stay & -1.046 & 0.199 & 0.000 & 0.351 & $(0.238,0.519)$ \\
\hline SICU readmission & 0.963 & 0.258 & 0.000 & 2.62 & $(1.579,4.347)$ \\
\hline Mechanical ventilation & 2.413 & 0.279 & 0.000 & 11.163 & $(6.461,19.290)$ \\
\hline CRRT & 0.888 & 0.243 & 0.000 & 2.43 & $(1.508,3.916)$ \\
\hline IL1 & 0.412 & 0.077 & 0.000 & 1.51 & $(1.299,1.754)$ \\
\hline IL2 & & & & & \\
\hline SICU surgical intensive care & unit, TNF-a tumor necrosis factor-alpha, IL interleukin, CRRT continuous \\
\hline renal replacement therapy & & & & & \\
\hline
\end{tabular}

Cytokine levels were increased in SICU readmission patients Of the 5,257 patients, 196 patients were readmitted to the SICU, giving a rate of $3.7 \%$. In the readmitted group, the length of SICU stay and length of mechanical ventilation were much longer than those in the other group; the rates of mechanical ventilation, CRRT, tracheotomy and emergency surgery were much higher in patients who were readmitted 
than those in patients who were not readmitted. According to the department, the highest readmission rate occurred in the general surgery department (5.0\%).

To determine whether cytokine levels were associated with the SICU readmission rate, we assessed the difference between the two groups. The readmission group patients had increased levels of IL-1 $\beta$, IL-2, IL6, IL-8, IL-10, TNF-a and procalcitonin (Table 4). Univariate and multivariate analyses showed that mechanical ventilation and the level of IL-2 could significantly influence SICU readmission, but no correlation was noted between IL-1 $\beta$, IL-6, IL-8, IL-10, TNF-a and procalcitonin levels and SICU readmission in the patients (Table 5). 
Table 4

Cytokine levels in patients with or without SICU readmission $(n=5,257)$

\begin{tabular}{|c|c|c|c|c|}
\hline \multirow[t]{2}{*}{ Variables } & \multicolumn{2}{|c|}{ SICU readmission $(n=5,257)$} & \multirow[t]{2}{*}{$z / x^{2}$} & \multirow[t]{2}{*}{$P$ value } \\
\hline & Yes $(n=196)$ & No $(n=5,061)$ & & \\
\hline Age (years) & $65.4 \pm 16.8$ & $62.2 \pm 16.3$ & -1.698 & 0.089 \\
\hline \multicolumn{3}{|l|}{ Sex, No. (\%) } & \multirow[t]{3}{*}{0.366} & \multirow[t]{3}{*}{0.545} \\
\hline Male & $121(3.9 \%)$ & $3015(96.1 \%)$ & & \\
\hline Female & $75(3.5 \%)$ & $2046(96.5 \%)$ & & \\
\hline Length of SICU stay (h) & $111.4 \pm 142.6$ & $62.7 \pm 115.3$ & -6.999 & 0.000 \\
\hline \multicolumn{3}{|l|}{ Length of SICU stay } & \multirow[t]{3}{*}{52.437} & \multirow[t]{3}{*}{0.000} \\
\hline$>7$ days & $37(10.9 \%)$ & $301(89.1 \%)$ & & \\
\hline$\leq 7$ days & $159(3.2 \%)$ & $4,760(96.8 \%)$ & & \\
\hline Length of mechanical ventilation (h) & $363.1 \pm 509.4$ & $124.2 \pm 712.2$ & -9.013 & 0.000 \\
\hline \multicolumn{3}{|l|}{ Mechanical ventilation } & \multirow[t]{3}{*}{154.379} & \multirow[t]{3}{*}{0.000} \\
\hline Yes & $118(9.6 \%)$ & $1,110(90.4 \%)$ & & \\
\hline No & $78(1.9 \%)$ & $3,951(98.1 \%)$ & & \\
\hline \multicolumn{5}{|l|}{ Tracheotomy } \\
\hline Yes & $45(17.9 \%)$ & $207(82.1 \%)$ & \multirow[t]{2}{*}{147.202} & \multirow[t]{2}{*}{0.000} \\
\hline No & $151(3.0 \%)$ & $4,854(97.0 \%)$ & & \\
\hline Length of CRRT & $120.3 \pm 84.7$ & $68.3 \pm 91.4$ & -3.854 & 0.000 \\
\hline \multicolumn{3}{|l|}{ CRRT } & \multirow[t]{3}{*}{43.814} & \multirow[t]{3}{*}{0.000} \\
\hline Yes & $23(13.0 \%)$ & $154(87.0 \%)$ & & \\
\hline No & $173(3.4 \%)$ & $4,907(96.6 \%)$ & & \\
\hline \multicolumn{5}{|l|}{ Emergency surgery } \\
\hline Yes & $86(5.7 \%)$ & $1,433(94.3 \%)$ & \multirow[t]{3}{*}{21.965} & \multirow[t]{3}{*}{0.000} \\
\hline No & $110(3.0 \%)$ & $3,613(97.0 \%)$ & & \\
\hline Not known & $0(0 \%)$ & $15(100 \%)$ & & \\
\hline Department & & & 9.365 & 0.025 \\
\hline $\begin{array}{l}\text { SICU surgical intensive care unit, TNF } \\
\text { renal replacement therapy }\end{array}$ & nor necrosis $f_{c}$ & r-alpha, IL inter & in, CRRT & tinuous \\
\hline
\end{tabular}




\begin{tabular}{|c|c|c|c|c|}
\hline \multirow[t]{2}{*}{ Variables } & \multicolumn{2}{|c|}{ SICU readmission $(n=5,257)$} & \multirow[t]{2}{*}{$z / x^{2}$} & \multirow[t]{2}{*}{$P$ value } \\
\hline & Yes $(n=196)$ & No $(n=5,061)$ & & \\
\hline General surgery & $62(5.0 \%)$ & $1,166(95.0 \%)$ & & \\
\hline Thoracic surgery & $38(3.0 \%)$ & $1,235(97.0 \%)$ & & \\
\hline Neurosurgery & $31(3.9 \%)$ & $767(96.1 \%)$ & & \\
\hline Endovascular surgery & $20(3.6 \%)$ & $538(96.4 \%)$ & & \\
\hline Orthopedics & $11(2.6 \%)$ & $406(97.4 \%)$ & & \\
\hline Urologic surgery & $10(3.2 \%)$ & $307(96.8 \%)$ & & \\
\hline Others & $24(3.6 \%)$ & $642(96.4 \%)$ & & \\
\hline $\mathrm{IL}-1 \beta(\mathrm{pg} / \mathrm{ml})$ & & & -3.775 & 0.000 \\
\hline Normal $(\leq 5)$ & $176(3.5 \%)$ & $4,878(96.5 \%)$ & & \\
\hline $5 \sim 10$ & $12(8.7 \%)$ & $126(91.3 \%)$ & & \\
\hline$\geq 10$ & $8(12.3 \%)$ & $57(87.7 \%)$ & & \\
\hline IL-2 (U/ml) & & & -7.427 & 0.000 \\
\hline Normal (223-710) & $76(2.3 \%)$ & $3,236(97.7 \%)$ & & \\
\hline $710-1,000$ & $47(5.2 \%)$ & $860(94.8 \%)$ & & \\
\hline $1,000-2,000$ & $48(6.2 \%)$ & 727 (93.8\%) & & \\
\hline $2,000-3,000$ & $10(6.0 \%)$ & $156(94.0 \%)$ & & \\
\hline$\geq 3,000$ & $15(15.5 \%)$ & $82(84.5 \%)$ & & \\
\hline IL-6 (pg/ml) & & & -5.592 & 0.000 \\
\hline Normal $(<3.4)$ & $10(2.6 \%)$ & $380(97.4 \%)$ & & \\
\hline $3.4-50$ & $78(2.9 \%)$ & $2,724(97.1 \%)$ & & \\
\hline $50-100$ & $30(3.8 \%)$ & $761(96.2 \%)$ & & \\
\hline $100-200$ & $29(4.8 \%)$ & 571 (95.2\%) & & \\
\hline$\geq 200$ & $49(7.3 \%)$ & 625 (92.7\%) & & \\
\hline IL-8 (pg/ml) & & & -3.261 & 0.001 \\
\hline Normal $(<62)$ & 148 (3.3\%) & 4,317 (96.7\%) & & \\
\hline
\end{tabular}




\begin{tabular}{|c|c|c|c|c|}
\hline \multirow[t]{2}{*}{ Variables } & \multicolumn{2}{|c|}{ SICU readmission $(n=5,257)$} & \multirow[t]{2}{*}{$z / x^{2}$} & \multirow[t]{2}{*}{$P$ value } \\
\hline & Yes $(n=196)$ & No $(n=5,061)$ & & \\
\hline $62-100$ & $22(6.9 \%)$ & $295(93.1 \%)$ & & \\
\hline $100-150$ & $8(4.7 \%)$ & $164(95.3 \%)$ & & \\
\hline $150-200$ & $5(5.2 \%)$ & $92(94.8 \%)$ & & \\
\hline$\geq 200$ & $13(6.3 \%)$ & $193(93.7 \%)$ & & \\
\hline IL-10 (pg/ml) & & & -4.357 & 0.000 \\
\hline Normal $(<9.1)$ & $125(3.1 \%)$ & $3,948(96.9 \%)$ & & \\
\hline $9.1-20$ & $41(5.9 \%)$ & $659(94.1 \%)$ & & \\
\hline $20-50$ & $15(5.1 \%)$ & $282(94.9 \%)$ & & \\
\hline $50-100$ & $6(6.8 \%)$ & $82(93.2 \%)$ & & \\
\hline$\geq 100$ & $9(9.1 \%)$ & $90(90.9 \%)$ & & \\
\hline TNF-a (pg/ml) & & & -4.474 & 0.000 \\
\hline Normal $(<8.1)$ & $64(2.5 \%)$ & 2,487 (97.5\%) & & \\
\hline $8.1-15$ & $58(3.8 \%)$ & $1,481(96.2 \%)$ & & \\
\hline $15-30$ & $39(5.6 \%)$ & $657(94.4 \%)$ & & \\
\hline $30-50$ & $19(7.1 \%)$ & $248(92.9 \%)$ & & \\
\hline$\geq 50$ & $16(7.8 \%)$ & $188(92.2 \%)$ & & \\
\hline Procalcitonin (ng/ml) & & & -5.911 & 0.000 \\
\hline Normal $(<0.5)$ & $102(2.6 \%)$ & $3,766(97.4 \%)$ & & \\
\hline $0.5-2$ & $38(5.1 \%)$ & $712(94.9 \%)$ & & \\
\hline $2-5$ & $27(8.8 \%)$ & $281(91.2 \%)$ & & \\
\hline $5-10$ & $15(10.2 \%)$ & $132(89.8 \%)$ & & \\
\hline$\geq 10$ & $14(7.6 \%)$ & 170 (92.4\%) & & \\
\hline
\end{tabular}


Table 5

Analysis of cytokine levels in relation to SICU readmission of patients

\begin{tabular}{|llllll|}
\hline Variables & $\boldsymbol{\beta}$ & SE & P value & OR & 95\% Cl \\
\hline Univariate analysis & & & & & \\
\hline Sex & -0.065 & 0.178 & 0.717 & 0.937 & $(0.661,1.329)$ \\
\hline Length of SICU stay & -0.356 & 0.235 & 0.130 & 0.7 & $(0.442,1.111)$ \\
\hline Mechanical ventilation & 1.315 & 0.2 & 0.000 & 3.724 & $(2.518,5.508)$ \\
\hline CRRT & 0.385 & 0.306 & 0.208 & 1.47 & $(0.807,2.678)$ \\
\hline Department & 0.068 & 0.069 & 0.328 & 1.07 & $(0.934,1.226)$ \\
\hline IL1 & 0.28 & 0.177 & 0.113 & 1.324 & $(0.936,1.871)$ \\
\hline IL2 & 0.249 & 0.095 & 0.009 & 1.283 & $(1.065,1.545)$ \\
\hline IL6 & 0.159 & 0.082 & 0.052 & 1.172 & $(0.999,1.376)$ \\
\hline IL8 & -0.101 & 0.102 & 0.320 & 0.904 & $(0.740,1.103)$ \\
\hline IL10 & -0.17 & 0.112 & 0.128 & 0.844 & $(0.677,1.05)$ \\
\hline TNF-a & -0.057 & 0.107 & 0.596 & 0.945 & $(0.766,1.166)$ \\
\hline Procalcitonin & 0.018 & 0.084 & 0.835 & 1.018 & $(0.863,1.200)$ \\
\hline Multivariate analysis & & & & & \\
\hline Mechanical ventilation & 1.366 & 0.166 & 0.000 & 1.29 & $(1.134,1.467)$ \\
\hline IL2 & 0.254 & 0.066 & 0.000 & 3.921 & $(2.829,5.434)$ \\
\hline $\begin{array}{l}\text { SICU surgical intensive care unit, TNF-a tumor necrosis factor-alpha, IL interleukin, CRRT continuous } \\
\text { renal replacement therapy }\end{array}$ & & & & & \\
\hline
\end{tabular}

Correlation between cytokine levels in patients with SICU readmission and outcomes Among the 196 patients with SICU readmission, the cytokine levels of 187 patients readmission were tested and collected. Overall, 161 patients survived, and 26 patients died; the mortality rate was $13.9 \%$, which was much higher than that seen with the first SICU admission (3.0\%).

For the third SICU admission, 10 patients $(5.3 \%, 10 / 187)$ were admitted, of which 8 patients were female, accounting for $80.0 \%$. Seven patients survived, and 3 patients died, with a mortality rate of $30.0 \%(3 / 7)$, which was much higher than that of the first and second SICU admissions.

To determine the outcomes and risk factors, we assessed the difference between survivors and nonsurvivors. Nonsurvivors had increased levels of IL-2 and procalcitonin compared with survivors, but no correlation was noted between IL-1 $\beta$, IL-6, IL-8, IL-10 and TNF-a levels and outcomes in the patients. 
Differences between the two groups were noted in the variables of age, length of SICU stay, mechanical ventilation, CRRT and department (Table 6). 
Table 6

Outcomes and risk factors for patients readmitted to the SICU

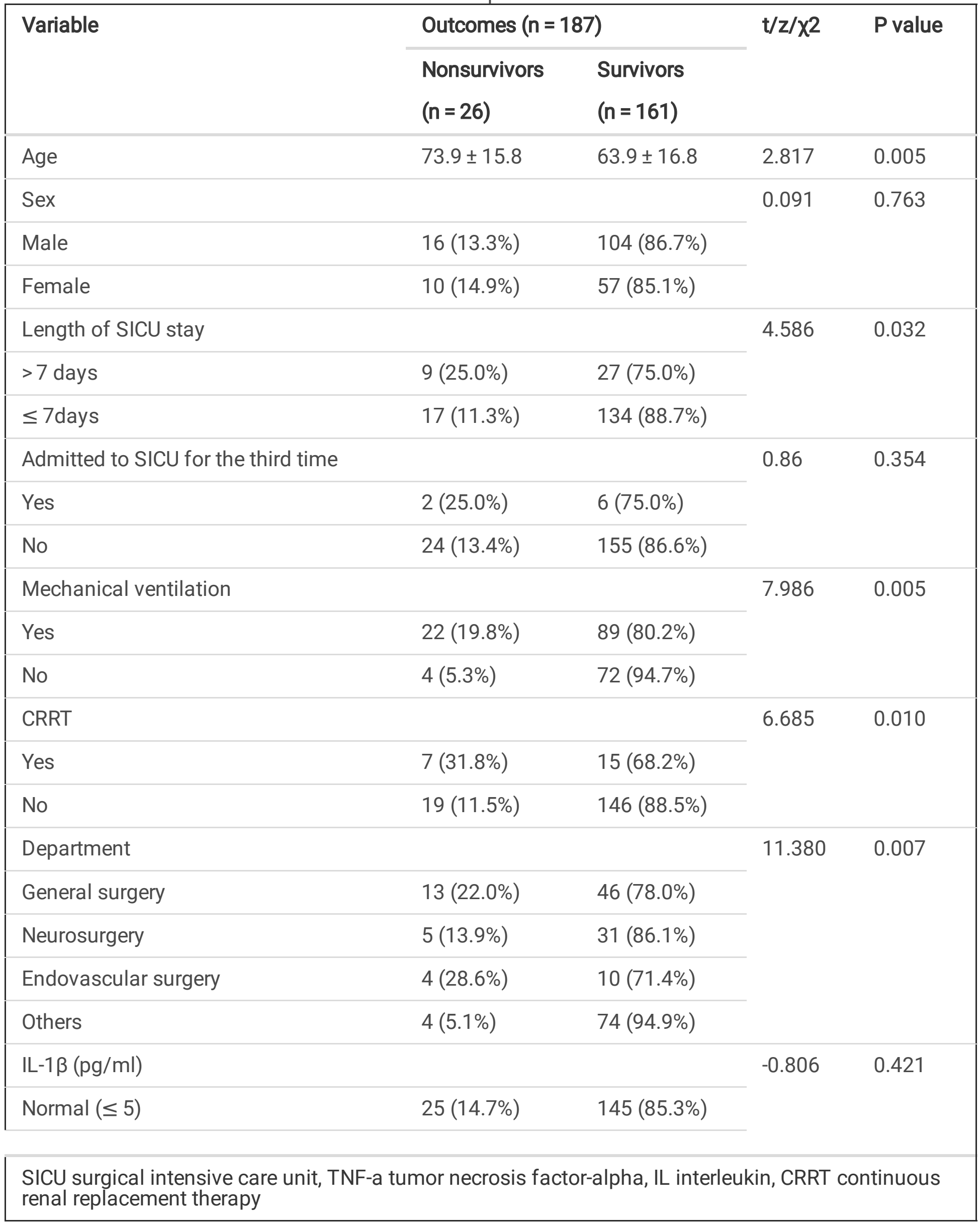




\begin{tabular}{|c|c|c|c|c|}
\hline \multirow[t]{3}{*}{ Variable } & \multicolumn{2}{|c|}{ Outcomes $(n=187)$} & \multirow[t]{3}{*}{$t / z / \chi^{2}$} & \multirow[t]{3}{*}{$P$ value } \\
\hline & Nonsurvivors & Survivors & & \\
\hline & $(n=26)$ & $(n=161)$ & & \\
\hline $5-10$ & $0(0 \%)$ & $9(100 \%)$ & & \\
\hline$\geq 10$ & $1(12.5 \%)$ & 7 (87.5\%) & & \\
\hline \multicolumn{3}{|l|}{ IL-2 (U/ml) } & \multirow[t]{6}{*}{-3.478} & \multirow[t]{6}{*}{0.001} \\
\hline Normal (223-710) & $5(5.8 \%)$ & $81(94.2 \%)$ & & \\
\hline $710-1,000$ & $3(9.4 \%)$ & 29 (90.6\%) & & \\
\hline $1,000-2,000$ & $11(23.4 \%)$ & $36(76.6 \%)$ & & \\
\hline $2,000-3,000$ & $3(30.0 \%)$ & $7(70.0 \%)$ & & \\
\hline$\geq 3,000$ & $4(33.3 \%)$ & $8(66.7 \%)$ & & \\
\hline \multicolumn{3}{|l|}{ IL-6 (pg/ml) } & \multirow[t]{6}{*}{-1.602} & \multirow[t]{6}{*}{0.109} \\
\hline Normal $(<3.4)$ & $0(0 \%)$ & $9(100 \%)$ & & \\
\hline $3.4-50$ & $8(10.7 \%)$ & $67(89.3 \%)$ & & \\
\hline $50-100$ & $5(17.9 \%)$ & $23(82.1 \%)$ & & \\
\hline $100-200$ & $4(13.8 \%)$ & 25 (86.2\%) & & \\
\hline$\geq 200$ & $9(19.6 \%)$ & 37 (80.4\%) & & \\
\hline \multicolumn{3}{|l|}{ IL-8 (pg/ml) } & \multirow[t]{6}{*}{-1} & \multirow[t]{6}{*}{0.317} \\
\hline Normal $(<62)$ & $18(12.7 \%)$ & $124(87.3 \%)$ & & \\
\hline $62-100$ & $4(19.0 \%)$ & $17(81.0 \%)$ & & \\
\hline $100-150$ & $1(12.5 \%)$ & 7 (87.5\%) & & \\
\hline $150-200$ & $0(0 \%)$ & $4(100 \%)$ & & \\
\hline$\geq 200$ & $3(25.0 \%)$ & $9(75.0 \%)$ & & \\
\hline \multicolumn{3}{|l|}{ IL-10 (pg/ml) } & \multirow[t]{4}{*}{-1.109} & \multirow[t]{4}{*}{0.268} \\
\hline Normal $(<9.1)$ & $16(13.2 \%)$ & $105(86.8 \%)$ & & \\
\hline $9.1-20$ & $2(5.1 \%)$ & 37 (94.9\%) & & \\
\hline $20-50$ & $3(21.4 \%)$ & $11(78.6 \%)$ & & \\
\hline
\end{tabular}




\begin{tabular}{|c|c|c|c|c|}
\hline \multirow[t]{3}{*}{ Variable } & \multicolumn{2}{|c|}{ Outcomes $(n=187)$} & \multirow[t]{3}{*}{$t / z / \chi^{2}$} & \multirow[t]{3}{*}{$P$ value } \\
\hline & Nonsurvivors & Survivors & & \\
\hline & $(n=26)$ & $(n=161)$ & & \\
\hline $50-100$ & $2(50.0 \%)$ & $2(50.0 \%)$ & & \\
\hline$\geq 100$ & $3(33.3 \%)$ & $6(66.7 \%)$ & & \\
\hline \multicolumn{3}{|l|}{ TNF-a (pg/ml) } & \multirow[t]{6}{*}{-1.273} & \multirow[t]{6}{*}{0.203} \\
\hline Normal $(<8.1)$ & $5(7.0 \%)$ & $66(93.0 \%)$ & & \\
\hline $8.1-15$ & $11(19.0 \%)$ & 47 (81.0\%) & & \\
\hline $15-30$ & $5(13.9 \%)$ & $31(86.1 \%)$ & & \\
\hline $30-50$ & $3(20.0 \%)$ & $12(80.0 \%)$ & & \\
\hline$\geq 50$ & $1(16.7 \%)$ & $5(83.3 \%)$ & & \\
\hline \multicolumn{3}{|l|}{ Procalcitonin (ng/ml) } & \multirow[t]{6}{*}{-2.031} & \multirow[t]{6}{*}{0.042} \\
\hline Normal $(<0.5)$ & $10(8.7 \%)$ & 105 (91.3\%) & & \\
\hline $0.5-2$ & $5(14.3 \%)$ & $30(85.7 \%)$ & & \\
\hline $2-5$ & $4(23.5 \%)$ & $13(76.5 \%)$ & & \\
\hline $5-10$ & $1(20.0 \%)$ & $4(80.0 \%)$ & & \\
\hline$\geq 10$ & $3(25.0 \%)$ & $9(75.0 \%)$ & & \\
\hline
\end{tabular}

The prognostic significance of IL-13, IL-2, IL-6, IL-8, IL-10, TNF-a and procalcitonin Overall 158 (3.0\%) of 5,257 patients died, and 5,099 (97.0\%) remained alive. The overall survival for patients with higher levels of IL-13, IL-2, IL-6, IL-8, IL-10, TNF-a and procalcitonin was significantly lower than that for patients with normal levels (Fig. 1) (Table 7). 
Table 7

Survival time of the patients

\begin{tabular}{|c|c|c|}
\hline Cytokines & & Survival time $(95 \% \mathrm{Cl})$ (days) \\
\hline \multicolumn{3}{|c|}{ IL-1 $\beta(p g / m l)$} \\
\hline & Normal $(\leq 5)$ & $57(40.004,73.996)$ \\
\hline & $5 \sim 10$ & $68(39.625,68.017)$ \\
\hline & $\geq 10$ & $28(13.122,28.360)$ \\
\hline \multicolumn{3}{|l|}{ IL-2 (U/ml) } \\
\hline & Normal (223-710) & $77(56.907,97.093)$ \\
\hline & $710-1,000$ & $41(16.838,65.162)$ \\
\hline & $1,000-2,000$ & $51(36.087,65.913)$ \\
\hline & $2,000-3,000$ & $63(14.28,111.72)$ \\
\hline & $\geq 3,000$ & $14(2.452,25.548)$ \\
\hline \multicolumn{3}{|l|}{ IL-6 (pg/ml) } \\
\hline & Normal $(<3.4)$ & $16(15.26,18.651)$ \\
\hline & $3.4-50$ & $77(43.82,110.18)$ \\
\hline & $50-100$ & $37(15.72,58.28)$ \\
\hline & $100-200$ & $28(15.495,40.505)$ \\
\hline & $\geq 200$ & $58(33.834,82.166)$ \\
\hline \multicolumn{3}{|l|}{ IL-8 (pg/ml) } \\
\hline & Normal $(<62)$ & $57(29.215,84.785)$ \\
\hline & $62-100$ & $41(9.561,72.439)$ \\
\hline & $100-150$ & $58(5.168,110.832)$ \\
\hline & $150-200$ & $19(15.876,22.702)$ \\
\hline & $\geq 200$ & $78(55.499,100.945)$ \\
\hline \multicolumn{3}{|c|}{$\mathrm{IL}-10$ (pg/ml) } \\
\hline & Normal $(<9.1)$ & $51(30.82,71.18)$ \\
\hline & $9.1-20$ & $47(36.221,142.982)$ \\
\hline & $20-50$ & $63(28.065,97.935)$ \\
\hline
\end{tabular}

TNF-a tumor necrosis factor-alpha, IL interleukin 


\begin{tabular}{|lll|}
\hline Cytokines & & Survival time $(95 \% \mathrm{Cl})$ (days) \\
\hline TNF-a $(\mathrm{pg} / \mathrm{ml})$ & $30-100$ & $26(1.411,68.589)$ \\
& & \\
& Normal $(<8.1)$ & $77(51.041,102.959)$ \\
\hline $8.1-15$ & $45(12.678,77.322)$ \\
\hline $15-30$ & $47(17.999,76.001)$ \\
\hline $30-50$ & $63(26.616,99.384)$ \\
\hline$\geq 50$ & $58(32.077,58.024)$ \\
\hline Procalcitonin $(\mathrm{ng} / \mathrm{ml})$ & & $66(29.409,102.591)$ \\
\hline Normal $(<0.5)$ & $58(23.514,92.486)$ \\
\hline $0.5-2$ & $25(5.611,55.102)$ \\
\hline $2-5$ & $51(27.84,74.16)$ \\
\hline $5-10$ & $37(2.289,71.711)$ \\
\hline$\geq 10$ & \\
\hline TNF-a tumor necrosis factor-alpha, IL interleukin \\
\hline
\end{tabular}

\section{Discussion}

Despite advances in supportive therapy and many clinical trials, among critically ill patients, adverse outcomes (sepsis, septic shock, organ dysfunction/failure, and mortality) remain high[11, 12]. Consequently, there is an urgent demand for better identification of variables for high-risk patients. At present, the determination of ICU patient risk mainly relies on clinical judgment and severity scores (such as APACHE scores), which incorporate patients' previous health statuses, along with clinical and laboratory variables, which track the function of multiple organ systems[13-16]. In recent decades, our knowledge of the biochemical processes underlying critical illness has considerably improved. We realized that cytokines perform important roles in host defense and maintenance of tissue homeostasis; however, abnormal or excessive production of cytokines disrupts these functions, resulting in inflammation and tissue injury[17], and there is strong evidence that cytokines work as important mediators of inflammatory and immunologic diseases and that elevated cytokine levels can contribute to organ dysfunction[18-20]. Evidence suggests that cytokines, including IL-8, TNF-a, and IL-10, strongly predict clinical outcome alone or in combination with other variables or widely accepted severity scores[21, 22]. 
Our study focused on critically ill patients after surgery, who received almost all possible surgeries in the SICU. For the first SICU admission, the mortality rate of the entire group was 3.0\% (158/5257), and the readmission rate was $3.7 \%(196 / 5,257)$. In agreement with other findings, we found higher levels of IL-1 $\beta$, IL-2, IL-6, IL-8, IL-10, TNF-a and procalcitonin in nonsurvivors than in survivors[17, 21]. Univariate and multivariate analyses showed that the influence of IL-1 $\beta(O R=1.855, P=0.000)$ and IL-2 $(O R=1.51, P=$ 0.000 ) on survival was significant. We also showed that cytokine levels correlated positively with the SICU readmission rate. Although the univariate and multivariate analyses only showed that the levels of $\mathrm{IL}-2(\mathrm{OR}=3.921, \mathrm{P}=0.000)$ could significantly influence the readmission rate, the SICU readmission rate was increased in patients with high levels of IL-1 $\beta$, IL-2, IL-6, IL-8, IL-10, TNF-a and procalcitonin compared with that in patients with normal levels. Our study revealed that in the entire population of critically ill patients after surgery, cytokines represent independent outcome predictors; in addition, IL-1 $\beta$ and IL-2 can determine mortality, and IL-2 can also influence the readmission rate.

Similar results were noted at SICU readmission. In total, 187 patients were included, including 161 survivors and 26 nonsurvivors, with a mortality rate of $13.9 \%$ (26/187), which was much higher than that for the first SICU admission (3.0\%). We still noted increased IL-2 levels in nonsurvivors ( $z=-3.478, p=$ $0.001)$. As a key cytokine involved in the immune response, IL-2 regulation can promote the differentiation of T cells and enhance the killing effect[3]. In a previous report, IL-2 levels were evaluated in the oral fluids of patients with hepatitis C or B and HIV[23]. Therefore, we can assume that IL-2 has a distinctive value for determining the state of local immunity in individuals, especially in critically ill patients. This has led to the development of novel therapeutic strategies using regulatory cytokines or cytokine-targeted biologics for the treatment of various diseases. According to some reports, the administration of low-dose IL-2 has resulted in promising results in treating autoimmune conditions such as chronic refractory graft-versus-host disease (GVHD), hepatitis $\mathrm{C}$ virus-induced vasculitis, and type 1 diabetes [24-26]. Some clinical trials have suggested that low-dose IL-2 might be effective in the treatment of systemic lupus erythematosus[27, 28].

We also analyzed patients who were admitted to the SICU for a third time. In total, 10 patients were included, including 2 males and 8 females and 3 nonsurvivors and 7 survivors, with a mortality of $30.0 \%$ $(3 / 10)$. We also noted increased IL-2 levels in nonsurvivors. As previously mentioned, we were able to demonstrate that older age, longer SICU stay, emergency surgery, high rate of mechanical ventilation and CRRT were associated with mortality and SICU readmission. One hypothesis is that these factors, including cytokine levels, correlate positively with the severity of critical illness and organ dysfunction. Regrettably, we omitted the data of the APACHE II score and could not analyze its association with outcomes. However, some studies have demonstrated that in nonseptic patients, cytokines represent independent outcome predictors along with disease severity scores, including APACHE II scores[4, 29]. However, other reports show contrasting results: cytokine levels cannot predict patient outcomes in nonseptic SICU patients[30,31]. Differences in patient condition (especially at diagnosis), the cytokine assays used and the data analysis methods used may explain the disparate results. 


\section{Limitations}

Potential limitations of this work include two points. First, we detected cytokines only on admission to the SICU. Sequential assessments of changes in cytokine levels may provide stronger evidence for the associations of the variables and outcomes. Second, we omitted the APACHE II score data, so the association between it, cytokine level and severity of disease could not be analyzed. Future studies should be undertaken to look at the association in critically ill patients after surgery.

\section{Conclusion}

In summary, the mortality rate increased with increasing number of admissions to the SICU $(3.0 \%<13.9 \%$ $<30.0 \%$ ). In critically ill patients after surgery, cytokine levels, including IL-1 $\beta$, IL-2, IL-6, IL-8, IL-10, TNF-a and procalcitonin levels, on admission to the SICU can predict outcome. Importantly, IL-2 seems to be the sole variable determining the outcome. Further studies are needed to explore the combined effect of cytokines and the sequential changes in cytokine levels in critically ill surgical patients.

\section{Abbreviations}

MOF

multiple organ failure; TNF-a:tumor necrosis factor-alpha; IL:interleukin; SICU:surgical intensive care unit; SIRS:systemic inflammatory response syndrome; CRRT:continuous renal replacement therapy; APACHE Il:acute physiology and chronic health evaluation; HIV:human immunodeficiency virus; GVHD:graftversus-host disease; IQRs:interquartile ranges; SDs:standard deviations; OR:odds ratio; Cl:confidence intervals.

\section{Declarations}

\section{Acknowledgements}

We would like to thank AJE (https://secure.aje.com/) for English language editing.

\section{Authors' contribution:}

Wenyan Pan and Yuxia Zhang conceived of this study. Yamin Yan collected the information, performed this study and drafted the article. Xiaorong Wang and Yan Hu participated in training the information collector and collecting the data. Zhenghong Yu and Yingjia Tang participated in check the quality of the collected data. Jingjing Li and Jinghua Mei participated in collecting and checking the patients' data. All authors have read and approved this article.

\section{Funding}


This work was supported by Youth Program of Zhongshan Hospital, Fudan University (no. 2019ZSQN01), Fuxing Nursing Program of Fudan University (no. FNF201945) andProgram of Fudan University "Double First-Class Discipline Construction"(no. 2018-40-22)".

\section{Availability of data and materials}

The datasets generated and/or analyzed during the current study are not publicly available due to ownership by the Department of SICU, Zhongshan Hospital, Fudan University, Shanghai, China, but are available from the corresponding author on reasonable request.

\section{Ethics approval and consent to participate}

The procedure mentioned in this study was in accordance with the standards of the Ethics Committee on Human Experimentation of Zhongshan Hospital, Fudan University(No. B2019-009). Informed consent from individual patients was waived because all data were anonymized for research purposes.

\section{Consent for publication}

Not applicable.

\section{Competing interests}

All the authors declare that they have no competing interests.

\section{References}

1. Ferreira FL, Bota DP, Bross A, Melot C, Vincent JL. Serial evaluation of the SOFA score to predict outcome in critically ill patients. JAMA. 2001;286:1754-8.

2. Oberholzer A, Oberholzer C, Moldawer LL. Cytokine signaling-regulation of the immune response in normal and critically ill states. Crit Care Med. 2000;28:N3-12.

3. Huang C, Zhou L, Chang X, Pang X, Zhang H, Zhang S. B7-H3, B7-H4, Foxp3 and IL-2 expression in cervical cancer: Associations with patient outcome and clinical significance. Oncol Rep. 2016;35:2183-90.

4. Dimopoulou I, Orfanos S, Kotanidou A, Livaditi O, Giamarellos-Bourboulis E, Athanasiou C, Korovesi I, Sotiropoulou C, Kopterides P, Ilias I, et al. Plasma pro- and anti-inflammatory cytokine levels and outcome prediction in unselected critically ill patients. Cytokine. 2008;41:263-7.

5. Netea MG, van der Meer JW, van Deuren M, Kullberg BJ. Proinflammatory cytokines and sepsis syndrome: not enough, or too much of a good thing? Trends Immunol. 2003;24:254-8. 
6. Shimazui T, Matsumura Y, Nakada TA, Oda S. Serum levels of interleukin-6 may predict organ dysfunction earlier than SOFA score. Acute Med Surg. 2017;4:255-61.

7. Quispe EA, Li XM, Yi H. Comparison and relationship of thyroid hormones, IL-6, IL-10 and albumin as mortality predictors in case-mix critically ill patients. Cytokine. 2016;81:94-100.

8. Gogos CA, Drosou E, Bassaris HP, Skoutelis A. Pro- versus anti-inflammatory cytokine profile in patients with severe sepsis: a marker for prognosis and future therapeutic options. J Infect Dis. 2000;181:176-80.

9. Sarbinowski R, Arvidsson S, Tylman M, Oresland T, Bengtsson A. Plasma concentration of procalcitonin and systemic inflammatory response syndrome after colorectal surgery. Acta Anaesthesiol Scand. 2005;49:191-6.

10. Dimopoulou I, Armaganidis A, Douka E, Mavrou I, Augustatou C, Kopterides P, Lyberopoulos P, Tzanela M, Orfanos SE, Pelekanou E, et al. Tumour necrosis factor-alpha (TNFalpha) and interleukin10 are crucial mediators in post-operative systemic inflammatory response and determine the occurrence of complications after major abdominal surgery. Cytokine. 2007;37:55-61.

11. Resche-Rigon M, Azoulay E, Chevret S. Evaluating mortality in intensive care units: contribution of competing risks analyses. Crit Care. 2006;10:R5.

12. Fatani SH, Al-Amodi AAAL, Kamel HS, Al-Khatieb HF, Bader K. H: Assessment of tumor necrosis factor alpha polymorphism TNF-alpha-238 (rs 361525) as a risk factor for development of acute kidney injury in critically ill patients. Mol Biol Rep. 2018;45:839-47.

13. Akavipat P, Thinkhamrop J, Thinkhamrop B, Sriraj W. Acute Physiology and Chronic Health Evaluation (Apache) li Score - the Clinical Predictor in Neurosurgical Intensive Care Unit. Acta Clin Croat. 2019;58:50-6.

14. Yalcin M, Godekmerdan E, Tayfur K, Yazman S, Urkmez M, Ata Y. The APACHE II Score as a Predictor of Mortality After Open Heart Surgery. Turk J Anaesthesiol Reanim. 2019;47:41-7.

15. Basile-Filho A, Lago AF, Menegueti MG, Nicolini EA, Rodrigues LAB, Nunes RS, Auxiliadora-Martins M, Ferez MA. The use of APACHE II, SOFA, SAPS 3, C-reactive protein/albumin ratio, and lactate to predict mortality of surgical critically ill patients: A retrospective cohort study. Med (Baltim). 2019;98:e16204.

16. Korkmaz Toker M, Gulleroglu A, Karabay AG, Bicer IG, Demiraran Y. SAPS III or APACHE IV: Which score to choose for acute trauma patients in intensive care unit? Ulus Travma Acil Cerrahi Derg. 2019;25:247-52.

17. Jordan SC, Choi J, Kim I, Wu G, Toyoda M, Shin B, Vo A. Interleukin-6, A Cytokine Critical to Mediation of Inflammation, Autoimmunity and Allograft Rejection: Therapeutic Implications of IL-6 Receptor Blockade. Transplantation. 2017;101:32-44.

18. Pinargote-Celorio H, Miralles G, Cano M, Caparros E, Portilla J, Gonzalez-Alcaide G, Ramos Rincon JM. Cytokine levels predict 30-day mortality in octogenarians and nonagenarians with communityacquired pneumonia: a retrospective observational study. Eur J Clin Microbiol Infect Dis. 2020;39:299-307. 
19. Landegger LD, Vasilijic S, Fujita T, Soares VY, Seist R, Xu L, Stankovic KM. Cytokine Levels in Inner Ear Fluid of Young and Aged Mice as Molecular Biomarkers of Noise-Induced Hearing Loss. Front Neurol. 2019;10:977.

20. Hardy-Werbin M, Rocha P, Arpi O, Taus A, Nonell L, Duran X, Villanueva X, Joseph-Pietras D, Nolan L, Danson $S$, et al. Serum cytokine levels as predictive biomarkers of benefit from ipilimumab in small cell lung cancer. Oncoimmunology. 2019;8:e1593810.

21. Heper Y, Akalin EH, Mistik R, Akgoz S, Tore O, Goral G, Oral B, Budak F, Helvaci S. Evaluation of serum C-reactive protein, procalcitonin, tumor necrosis factor alpha, and interleukin-10 levels as diagnostic and prognostic parameters in patients with community-acquired sepsis, severe sepsis, and septic shock. Eur J Clin Microbiol Infect Dis. 2006;25:481-91.

22. Livaditi O, Kotanidou A, Psarra A, Dimopoulou I, Sotiropoulou C, Augustatou K, Papasteriades C, Armaganidis A, Roussos C, Orfanos SE, Douzinas EE. Neutrophil CD64 expression and serum IL-8: sensitive early markers of severity and outcome in sepsis. Cytokine. 2006;36:283-90.

23. Azatyan V, Yessayan L, Shmavonyan M, Melik-Andreasyan G, Perikhanyan A, Porkshenyan K. Evaluation of IL-2, IL-10, IL-4 and -interferon levels in the oral fluids of patients with hepatitis C, B and HIV. J Infect Dev Ctries. 2019;13:69S-74S.

24. Tomova R, Antonov K, Ivanova A, Jacobs JJ, Koten JW, Den Otter W, Krastev Z. Low-dose IL-2 therapy reduces HCV RNA and HBV DNA: case report. Anticancer Res. 2009;29:5241-4.

25. Koreth J, Matsuoka K, Kim HT, McDonough SM, Bindra B, Alyea EP 3rd, Armand P, Cutler C, Ho VT, Treister NS, et al. Interleukin-2 and regulatory T cells in graft-versus-host disease. N Engl J Med. 2011;365:2055-66.

26. Hartemann A, Bensimon G, Payan CA, Jacqueminet S, Bourron O, Nicolas N, Fonfrede M, Rosenzwajg M, Bernard C, Klatzmann D. Low-dose interleukin 2 in patients with type 1 diabetes: a phase 1/2 randomised, double-blind, placebo-controlled trial. Lancet Diabetes Endocrinol. 2013;1:295-305.

27. He J, Zhang R, Shao M, Zhao X, Miao M, Chen J, Liu J, Zhang X, Jin Y, Wang Y, et al. Efficacy and safety of low-dose IL-2 in the treatment of systemic lupus erythematosus: a randomised, doubleblind, placebo-controlled trial. Ann Rheum Dis. 2020;79:141-9.

28. Ballesteros-Tato A, Papillion A. Mechanisms of action of low-dose IL-2 restoration therapies in SLE. Curr Opin Immunol. 2019;61:39-45.

29. Rodriguez-Gaspar M, Santolaria F, Jarque-Lopez A, Gonzalez-Reimers E, Milena A, de la Vega MJ, Rodriguez-Rodriguez E, Gomez-Sirvent JL. Prognostic value of cytokines in SIRS general medical patients. Cytokine. 2001;15:232-6.

30. Friedland JS, Porter JC, Daryanani S, Bland JM, Screaton NJ, Vesely MJ, Griffin GE, Bennett ED, Remick DG. Plasma proinflammatory cytokine concentrations, Acute Physiology and Chronic Health Evaluation (APACHE) III scores and survival in patients in an intensive care unit. Crit Care Med. 1996;24:1775-81.

31. Bonville DA, Parker TS, Levine DM, Gordon BR, Hydo LJ, Eachempati SR, Barie PS. The relationships of hypocholesterolemia to cytokine concentrations and mortality in critically ill patients with 
systemic inflammatory response syndrome. Surg Infect (Larchmt). 2004;5:39-49.

Figures
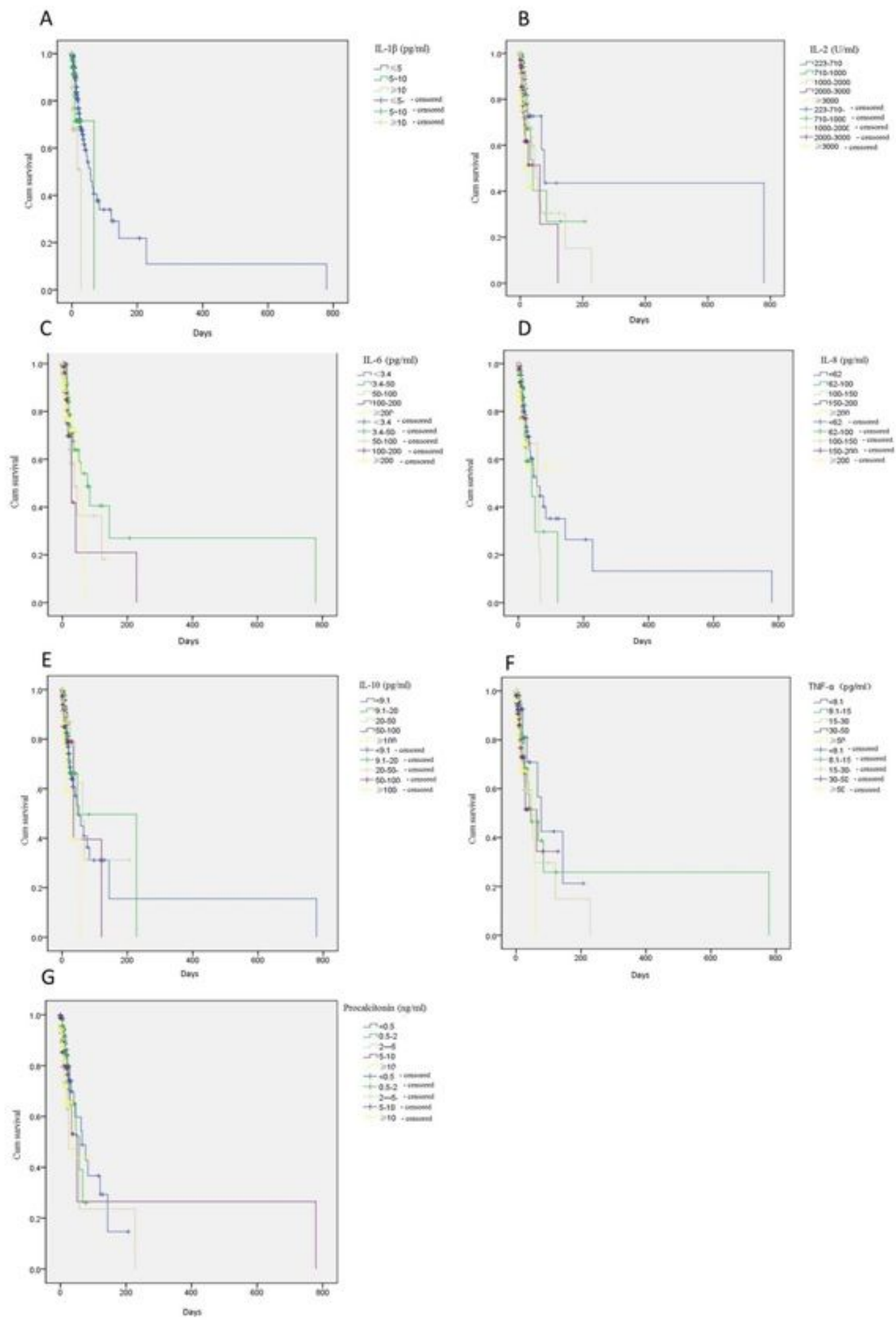

Figure 1 


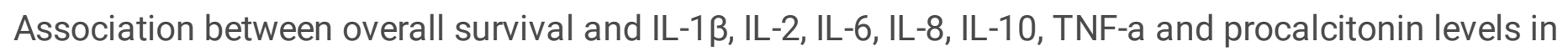
5,257 patients. (A-G) Patients with higher levels of IL-1 $\beta$, IL-2, IL-6, IL-8, IL-10, TNF-a and procalcitonin showed longer survival times than those with normal levels. 